\title{
Antecubital arteriovenous fistula in hemodialysis; a comparison between side-to-side versus end-to-side venous anastomosis
}

\author{
Iraj Nazari ${ }^{1}$, Seyed Mansour Alamshah ${ }^{1}$, Mohammad Bahadoram $^{2}$, Nozar Dorestan $^{1^{*}}$ \\ ${ }^{1}$ Department of Vascular Surgery, Faculty of Medicine, Ahvaz Jundishapur University of Medical Sciences, Ahvaz, Iran \\ ${ }^{2}$ Medical Students Research Committee, Ahvaz Jundishapur University of Medical Sciences, Ahvaz, Iran
}

\section{AR T I C L E I N F O}

Article Type:

Original

\section{Article History:}

Received: 12 March 2017

Accepted: 7 July 2017

Published online: 6 August 2017

\section{Keywords:}

Hemodialysis

Anastomosis

Arteriovenous fistula

Chronic kidney disease

End-stage renal disease

\begin{abstract}
A B S T R A C T
Introduction: Chronic Kidney Disease (CKD) is a global health problem which is growing in prevalence. Hemodialysis is the most common treatment for end-stage renal disease (ESRD) which requires a permanent vascular access. Vascular accesses should have a good patency and low complication.

Objectives: The present study aimed to compare side-to-side (STS) versus end-to-side (ETS) methods in antecubital arteriovenous fistulas in dialysis patients.

Patients and Methods: In this randomized, single-blind clinical trial, 100 ESRD patients who scheduled for hemodialysis were divided into two groups. STS arteriovenous anastomosis was employed in one group and in the other, the ETS approach was conducted. Follow ups were done after 1,8 , and 24 weeks to detect fistula maturation and immediate or delayed complications.

Results: The STS anastomosis group demonstrated higher rates of delayed maturation, vascular aneurism, and venous hypertension syndrome than ETS anastomosis group. The ETS group, however, showed higher rates of venous thrombosis and vascular stenosis. Overall, the rate of complications was $24 \%$ and $8 \%$ for STS and ETS anastomosis group respectively $(P=0.03)$.

Conclusion: In comparison, ETS arteriovenous fistulas involve less complications than STS method in ESRD patients. However, more thrombosis and stenosis detected by ETS method. ETS arteriovenous fistulas had less delayed maturation, aneurism, venous hypertension and overall complications than STS approach. ETS arteriovenous fistulas contributes to establishing a more stable connection to hemodialysis machine to conduct a more effective hemodialysis.
\end{abstract}

Implication for health policy/practice/research/medical education:

In a study on 100 end-stage renal disease, we found ETS arteriovenous fistulas had less complications compared to the STS technique in this patients.

Please cite this paper as: Nazari I, Alamshah SM, Bahadoram M, Dorestan N. Antecubital arteriovenous fistula in hemodialysis; a comparison between side-to-side versus end-to-side venous anastomosis. J Renal Inj Prev. 2017;6(4):282-285. DOI: $10.15171 /$ jrip.2017.53.

\section{Introduction}

Chronic kidney disease (CKD) is a worldwide health problem. CKD has an increasing rate due to an aging population and increasing of diabetes, hypertension and cardiovascular disease (1). Currently, there are more than one and a half billion CKD patients living worldwide whose proportion is expected to exceed in near future (2). Hemodialysis is encountered as the most common type of treatment for end-stage renal disease (ESRD) patients (3). Vascular accesses should provide an easy and acceptable flow, during hemodialysis. Arteriovenous fistula (AVF) is known as the best way for vascular access in dialysis $(4,5)$. AVF is created by anastomosis of an artery to a vein. This technique provides blood flow from deep arteries to surface veins. A $200-400 \mathrm{~mL} / \mathrm{min}$ blood flow is provided by cannulation of the surface veins which is required for 
an effective hemodialysis (6).

To date, various techniques and sites have been developed for constructing the fistula, among which the wrist and the antecubital fossa are the most suitable places $(7,8)$. According to national American society for vascular surgery, wrist is the first choice for radio-cephalic fistula. However, most patients do not have valid arteries in the mentioned site. Thus, the brachiocephalic fistula in the antecubital fossa is the next choice. The artery used for creating this fistula is usually the brachial artery but another option is to use the proximal part of the radial artery $(5,9,10)$

Three most common anastomosis approaches ESRD patients include end-to-end (ETE), end-to-side (ETS), and side-to-side (STS) methods. There is still dispute over which method is best for each patient. Several studies have demonstrated, no major differences is the patency rate of these methods in animal models.

Complications of vascular access are the morbidity of patients on hemodialysis. Furthermore, fistula dysfunction is one of the most common causes of secondary intervention and re-hospitalization in these patients. In fact, $25 \%$ of hospital admissions of dialysis patients are due to vascular access complications.

\section{Objectives}

Our study aimed to compare the STS and ETS anastomosis methods in arteriovenous antecubital fistula in dialysis patients.

\section{Patients and Methods}

Study design and setting

This single blind clinical trial was conducted on patients referred to Golestan hospital affiliated with Ahvaz Jundishapur University of Medical Sciences in a timeframe of 2 years. All patients entered into the study were antecubital fistula candidates.

Eligible patients randomly assigned to each group of STS and ETS anastomosis. There was no significant difference between the groups at the beginning of the study. Participants were assigned to one of the two groups of 50 patients. The first group underwent STS anastomosis and the second group went by ETS anastomosis.

One week after surgery, patients returned for the first follow up to check consistency of fistula. The next follow up was scheduled for 6-8 weeks later. Fistula maturation was determined by Doppler sonography. Patients were examined by Toshiba Nemio 30 color Doppler sonography using $10 \mathrm{MHz}$ linear probe in supine position on extended elbow. This examination revealed brachial artery diameter size and mean blood velocity per time unit of brachial blood flow. Moreover, brachial blood flow volume was calculated by cc/min. Accordingly fistula blood flow volume was determined by color Doppler sonography too. Fistula was considered mature if the venous diameter was about $6 \mathrm{~mm}$. The skin-fistula distance was also $6 \mathrm{~mm}$, and the flow was more than $600 \mathrm{cc} / \mathrm{min}$. If the fistula was not matured during this period, patients will return every 2 weeks for another sonography until the date of delayed fistula maturation was established. All patients were followed up for 6 months after the procedure for consideration of probable complications.

\section{Assessments}

Doppler sonography and physical examinations were applied for assessment of complications. Fistula aneurism was defined by localized inflation of the vein with a diameter of 1.5 times the size of a healthy fistula segment. Skin thinness or injuries, scars, or spontaneous bleedings were recorded during the examinations. Steal syndrome was determined as an immediate or late complication determined by severe muscle weakness, sensory weakness, chill and also discoloration of the skin of the hand and pain that intensified during dialysis. Diagnosis of venous hypertension syndrome was made by observing various clinical symptoms such as pitting edema, redness, warmness, and heaviness of the limb in acute conditions and lasting hand edema and distal extremity ulcers in chronic conditions. Thrombosis was diagnosed by absence of thrill and bruit and impalpable distal pulse. Stenosis was determined by a bruit at a narrowed segment (high pitch) which could only be heard during the systolic time. In such cases, fistula output was reduced and dialysis conducted with a decreased speed and volume. This was a sign that the fistula needed attention.

\section{Ethical issues}

The study protocol was approved by Ethical Committee of Ahvaz Jundishapur University of Medical Sciences (\#U93103). The research followed the tenets of the Declaration of Helsinki. Written informed consent was obtained from all patients and remained confidential.

\section{Statistical analysis}

Data analysis was conducted by SPSS software version 17. First, descriptive analysis including distribution tables, charts and central tendency measures (mode, median, mean \pm SD) were defined. Chi-squared test was applied to examine the relationship between qualitative variables. The significance level was set at $P<0.05$.

Results

The analysis compared the result of STS and ETS anastomosis methods in antecubital arteriovenous fistulas in 100 patients requiring dialysis due to ESRD ( 48 females and 52 males). Mean age of females was 44 and males was 56. Two patients died during the study and were excluded from analysis. All fistulas were open in the first week of examination. The 6-week follow up showed that STS anastomosis patients had delayed maturation (16\%), thrombosis (2\%), aneurism (6\%), stenosis (2\%), and venous hypertension syndrome (12\%). Overall, $24 \%$ of the patients showed complications. Only one patient (2\%) with steal syndrome was observed.

The ETS anastomosis group showed delayed maturation $(4 \%)$, thrombosis $(12 \%)$, aneurism $(2 \%)$, stenosis $(4 \%)$, 
and venous hypertension syndrome (2\%). Overall, $8 \%$ complication rate was reported.

Delayed maturation is seen in both groups of STS and ETS. The frequency in STS (16\%) is higher than ETS (4\%) which is statistically significant $(P=0.047)$. The rate of vascular thrombosis in STS was $2 \%$ while in ETS, it was $12 \%$. This finding shows a significant difference with a much higher rate in ETS $(P=0.05)$. The incidence of aneurism for STS group was $6 \%$ and $2 \%$ for ETS group $(P$ $=0.31)$. Stenosis rate at the anastomosis site was reported $2 \%$ for STS group and $4 \%$ for ETS group $(P=0.56)$. The frequency rate of venous hypertension syndrome in STS group was $12 \%$ and for ETS group was $2 \%$ which was statistically significant. Overall, the rate of complications was $24 \%$ and $8 \%$ for STS and ETS anastomosis group respectively $(P=0.03)$. This finding shows, complications rate of ETS anastomosis was lower than STS anastomosis group $(P=0.03)$.

\section{Discussion}

During a 2-year study, arteriovenous anastomosis was performed on 100 ESRD patients, candidate for antecubital arteriovenous fistula by either STS or ETS methods or complications and outcomes of both approaches were compared.

Galić et al investigated 260 hemodialysis patients based on relationship between complication onset and the anastomosis method in their surgery (9). The complication rate was $6.15 \%$ and $7.31 \%$ in ETS and STS, respectively. The thrombosis rate was $2.31 \%$ in ETS and $5.56 \%$ in STS. The rate for primary dilation (over a 6-month-period after fistula fixation) was $10.67 \%$ in ETS and $18.88 \%$ in STS. The rate for secondary dilation (fistula performance maintenance 24 months after fixation) was $89.23 \%$ in ETS and $81.11 \%$ in STS. In our study, delayed maturation in STS group was $16 \%$ and $4 \%$ in ETS group which shows a significant difference. The study conducted by Galić et al, also showed a lower rate of delayed maturation in ETS (11\%) than STS (16\%), which was similar to our results. However, the difference was not statistically significant. They concluded, anastomosis by STS technique has better outcome both in duration and maintenance as well as fewer complications compared to other arteriovenous fistula construction methods (9).

Similarly, arteriovenous fistula placement in patients who required hemodialysis by end-to-size anastomosis method had less complications such as delayed maturation, aneurysm and venous hypertension. These factors are important for an effective hemodialysis. However, more thrombosis and stenosis complications have been reported by this method.

In a study by Stanziale et al, ETE in comparison to ETS has more late thrombosis (10\% vs $4.1 \%$ ) and stenosis $(21.4 \%$ vs $2.7 \%$ ). However, early thrombosis was similar in both groups. Primary patency was $80 \%$ for the first group and $85.7 \%$ for the second group during the first year. Although the difference was not statistically significant. They concluded, anastomosis by ETS method has better outcome (11).

Contrary to our study, Zarin et al investigated 190 patients with ESRD whose arteriovenous fistulas were created by either ETS or STS methods. Furthermore, they compared the operation time, immediate success and short-term complications. The mean operation time for STS group was 50 and 75 minutes for ETS (12). Bleeding required reexploration in 4 cases $(5.7 \%)$ in ETS group and 2 (1.7\%) in STS group. The immediate failure rate of the procedure was $2.5 \%$ for STS group and $7.5 \%$ for ETS group. Wound infection occurred in one case (1.4\%) of ETS group and 2 cases $(1.7 \%)$ in STS group. They concluded that creating arteriovenous anastomosis by STS method requires shorter time and has fewer complications than end-to-end method.

Likewise, in the study by Nouri et al, the efficacy of arteriovenous fistula of STS and ETS methods were compared in patients with ESRD. The comparison was based on fistula efficacy, breakdown rate and venous hypertension. Around 30 cases were evaluated in each group. There were six cases $(20 \%)$ of breakdown in STS group, where four cases were due to fistula thrombosis and two cases had occurred due to venous hypertension, and therefore the fistula was ligated. There were five cases (16.6\%) of fistula failure in ETS group, all of them were due to thrombosis. They detected, the efficacy rate of arteriovenous fistula did not have a significant difference in the two groups during the six months evaluation (13).

\section{Conclusion}

It seems that the ETS arteriovenous fistula construction carries less complications compared to STS approach in ESRD patients who require hemodialysis. Although thrombosis and stenosis are often reported in ETS method however, it comprises less delayed maturation, aneurism, venous hypertension, and lower overall complications rate which are essential for a successful dialysis.

\section{Limitations of the study}

The most important limitation of the study was its small sample size. We suggest larger studies on this topic in hemodialysis patients.

\section{Acknowledgments}

We sincerely appreciate the collaboration of vascular surgery section and hemodialysis unit's staffs of Golestan hospital.

\section{Authors' contribution}

Study concept and design; SA and MB. Acquisition of data; SMA and IN. Analysis and interpretation of data: MB. Drafting of the manuscript; ND. Critical revision of the manuscript for important intellectual content: ND, SMA and IN. Statistical analysis: MB. Administrative, technical, and material support; ND, SMA. Study supervision; SMA and IN.

\section{Conflicts of interest}

The authors declare that they have no competing interests. 


\section{Ethical considerations}

Ethical issues (including plagiarism, data fabrication, double publication) have been completely observed by the authors.

\section{Funding/Support}

This study was funded by vice chancellor for research and technology of Ahvaz Jundishapur University of Medical Sciences, Ahvaz, Iran (Grant\# U-93103).

\section{References}

1. Modaresi A, Nafar M, Sahraei Z. Oxidative stress in chronic kidney disease. Iran J Kidney Dis. 2015;9:165-79.

2. Yoo DW Yoon M, Jun HJ. Successful access rate and risk factor of vascular access surgery in arm for dialysis. Vasc Specialist Int. 2014;30:33-7. doi: 10.5758/vsi.2014.30.1.33.

3. Twardowski ZJ. History of hemodialyzers' designs. Hemodial Int. 2008;12:173-210. doi: 10.1111/j.15424758.2008.00253.x.

4. Sidawy AN, Spergel LM, Besarab A, Allon M, Jennings WC, Padberg FT Jr, et al. The Society for Vascular Surgery: clinical practice guidelines for the surgical placement and maintenance of arteriovenous hemodialysis access. J Vasc Surg. 2008;48:2S-25S. doi: 10.1016/j.jvs.2008.08.042.

5. Marcus RJ, Marcus DA, Sureshkumar KK, Hussain SM, McGill RL. Gender differences in vascular access in hemodialysis patients in the United States: developing strategies for improving access outcome. Gend Med. 2007;4:193-204.
6. Agarwal AK. Systemic effects of hemodialysis access. Adv Chronic Kidney Dis. 2015;22:459-65. doi: 10.1053/j. ackd.2015.07.003.

7. Hashemi H, Sheridan MJ, Ford B. Selective two-stage basilic and cephalic vein transpositions can significantly improve the rate of fistula construction. Int J Angiol. 2009;18:12934.

8. Baktiroglu S, Yanar F, Ozturk A. Brachiocephalic and basilic fistula. J Vasc Access. 2015; 16:S29-33. doi: 10.5301/ jva.5000377.

9. Galić G, Kvesić A, Tomić M, Rebac Z, Brekalo Z, Martinović $\mathrm{V}$, et al. The advantages of end-to-side arteriovenous anastomosis over the other two types of arteriovenous anastomosis in dialysis patients. Coll Antropol. 2008;32:109-14.

10. Lynn KL, Buttimore AL, Wells JE, Inkster JA, Roake JA, Morton JB. Long-term survival of arteriovenous fistulas in home hemodialysis patients. Kidney Int. 2004;65:1890-6.

11. Stanziale R, Lodi M, D'Andrea E, Sammartino F, DI Luzio V. Arteriovenous fistula: end-to-end or end-to side anastomosis? Hemodial Int. 2011;15:100-3. doi: 10.1111/j.1542-4758.2010.00503.x.

12. Zarin M, Ahmad I, Waheed D, Zeb A, Anwar N, Aslam $\mathrm{V}$, et al. Arteriovenous fistula construction in chronic haemodialysis patients: comparison of end-to-side and side-to-side techniques. J Coll Physicians Surg Pak. 2004;14:619-21.

13. Nouri A, Ahmadi A. The efficacy of arteriovenous fistula of end to end and end to side in chronic renal failure patients referring for hemodialysis. Pejouhandeh. 2002;7:25-28.

Copyright (c) 2017 The Author(s); Published by Nickan Research Institute. This is an open-access article distributed under the terms of the Creative Commons Attribution License (http://creativecommons.org/licenses/by/4.0), which permits unrestricted use, distribution, and reproduction in any medium, provided the original work is properly cited. 